\title{
A METHOD AFFORDING DENTURE SERVICE IMMEDIATELY FOLLOWING EXTRACTION
}

\author{
By ROBERT R. GILLIS, D.D.S., Hammond, Indiana
}

(Read before the National Society of Denture Prosthetists, August 20, 1920, and the National Dental Association, Boston, Massachusetts, August 23-27, 1920)

W E FREQUENTLY meet with patients for whom it would be highly desirable to provide dentures immediately after we have extracted their natural teeth.

Such patients by reason of their pride in personal appearance-their reluctance "to be seen without teeth"-are willing to pay for the extra service involved in supplying them with dentures during that intervening period between the time of extraction and the time when the alveolar ridges are sufficiently dissolved when we may reasonably expect more permanency in the fit.

Men and women alike of ten make a desperate effort to retain badly diseased natural teeth just because they dread appearing "toothless" among their business and social associates.

In the light of our present knowledge concerning the subtle evils of oral foci of infection, our present effort is made with the hope that we may thereby remove the patient's last argument for the retention of diseased teeth.

The man of common labor or the shopkeeper whose work compels him to appear daily before his customers and converse with them; the woman whose duties in an office or schoolroom afford no leisure time during which she can go into retirement while waiting for teeth; all busy people, as well as those persons of leisure whose social duties only are most to be consulted, appreciate our efforts to keep them dentally presentable.
In these modern times of stress and strife, "strep and staph" have created a condition that will compel a revision of Skakespeare's Seven Ages to the extent that the one qualification "sans teeth" applied to his seventh age should be moved forward several lines and placed just after "sighing like a furnace"-for I can imagine nothing that makes a person sigh more than to be told he must loose his teeth.

Thru the method herein offered, we would make possible one further revision of Shakespeare's classic by eliminating entirely the words "sans teeth." Nobody now needs to appear sans teeth at any stage of life.

Nothing original is claimed for the system here described.

We have selected the several steps in procedure from various methods and tried to assemble a technic that is adaptable to all cases.

For many years, a few dentists, having extracted all the molars and bicuspids previously, have aimed at like results by making impressions of the ridges and teeth and registering central occlusion before extraction of the front teeth; recovering the casts; trimming off the teeth; then setting up and vulcanizing dentures over these modified casts. That method is accurate only to such degree as the operator is a good guesser for he must trim the gingival contours by guess only.

Where pyorrhea has caused consider- 
able recession of the gums, such an approximate method may succeed fairly well.

Or, in a case presenting normal gum and ridge conditions, fair success may be attained by grinding the necks of the front teeth to fit the ridge and not attempting to make a labial rim of vulcanite on the denture. We know from experience that dentures made without labial rims do not afford the same degree of efficiency as those made with complete rims.

The method herein offered makes it possible for us to construct a denture within a few hours after extraction that will afford greater efficiency than is possible from the older methods of making a denture from impressions made before extraction.

\section{Preliminary Study of Case}

When the prospective denture patient first appears, we should immediately begin a close examination of conditions, studying the patient's features to determine all the facial peculiarities.

In talking with the patient, note the variations of action in the two sides of the face as to the movements of the muscles of expression and mastication. You will be surprised to observe the asymetrical conditions in most adults.

Observe to what degree the natural gums are displayed when the patient smiles and laughs; note the position and contour of the lips when the mandible is at rest; the length of the teeth in relation to the lip lines; and any peculiarly atypical arrangement of the teeth. Ail these observations are to be made while the patient is entirely unconscious of your close scrutiny.

The writer has found no freld in dental service wherein the patient and family and friends are so free to offer criticism as in denture service.

For this very reason, in anticipation of criticism, to forestall any unjust criticism, and to avoid future misunderstandings, we have found it to be to the mutual advantage of patient and operator to reach a thoro understanding on several points before the natural teeth are disturbed.

When it is agreed and determined that we are to go thru with the case, we make and record some measurements and make some impressions from which casts will be made for future reference.

\section{Measurements}

With a metric rule, measure the distance from the angle where the nose and lip meet in the median line to the incisal of the maxillary central. It is well also to measure the distance from the angle at the alae of the nose to the cuspid incisals.

With these measurements, you will be able to restore these dimensions in your denture-or make such variations as have been agreed upon previously.

Now measure in the median line from the nose-lip angle to the lower border of the chin; in this dimension, make two records, first with the teeth in central occlusion and second with the mandible at rest. There will be a variance of from 1 to 6 millimeters between these two positions for with the mandible at rest, the teeth are not in occlusion.

These measurements enable us to reestablish central occlusion for the case insuring against making the occlusal rims either too long or too short.

In those cases where the natural teeth are much in evidence, in smiling or laughing, we measure also the width and heighth of the exposed gum area. This record should be made also upon one of the reference casts of this case.

All these measurements should be recorded for future reference.

We next pass a smàll straightedge or bar across the maxillary teeth just back of the cuspids and ask the patient to close the mandibular teeth against the bar so as to hold it steadily in place while we observe whether it is parallel to a line drawn thru the pupils of the patient's eyes. Any variance should be 
noted. This bar should be parallel with the eye-line. If any variance is evident, call the patient's attention to it at this time.

Our next step is to make a record of the facial contour along the median line. At first we used a piece of 12 gauge leadfuse wire; but this proved rather soft and too easily altered so we now employ a 14 gauge aluminum wire.

Straighten out a piece of wire 12 inches long. Holding one end on the head in the median line just above the hair line, carry the wire under pressure against the forehead, down between the eyes and to the tip of the nose. Bending the wire with the fingers or suitable pliers, conform it to the tip of the nose, lips and chin.

When finished this wire contour gauge, rested lightly along the median line of the face, should be in contact thruout its length but not impinging or depressing the tissues at any point. This wire is then laid upon a piece of bristol-board and its shape marked along the inside with a pencil. Cut carefully along the mark and the result will be a silhoutte of our patient on the one side and the cutout portion will serve as our contour guide for future reference.

This card also becomes the proper place on which to record all the other data bearing upon the case.

A second contour gauge may be likewise shaped with wire beginning at the lobe of one ear passing forward on the face on a level with the fullest contour of the upper lip and terminating at the lobe of the opposite ear; still another transverse guide may be indicated in some cases, passing from ear to ear just below the alae of the nose.

The measurements thus far made will enable us to locate the artificial central so that its incisal shall be a definite distance from the nose- lip angle in reproduction of the natural dimensions. How may we be able to locate it definitely anterio-posteriorly in the sagittal plane or median plane so that it cannot be too far forward or too far back as judged by the location of the natural central?

Our profile guide solves this problem. Adjust it to the face; hold the metric rule in such a way that one corner touches the median incisal of the central incisor while the rule lies against the cardboard. Maintain this position while a line is drawn across the end of the rule and down along the side. This line is our landmark to which we may come back any time with the same rule and thus re-establish by its inner corner the exact location of the natural central. Likewise it gives us a means of checking up when setting the porcelain teeth so there can be no error where duplication is sought in position.

The use of this profile guide alone probably eliminates more guesswork on the dentist's part and more complaint from the patient than any other feature of this whole system.

\section{PRELIMINARY IMPRESSIONS}

We now take a small roll of softened modeling compound; have the patient shut the teeth in central occlusion, relaxing the lips; place the compound over the front teeth, (covering about to second bicuspid on each side), bring the lips over the compound and then with a napkin over the lips press and burnish the compound against the teeth and gums. Remove when stone cold and you have an impression from which you will secure a cast-relief of the labial appearance of this patient's teeth.

The next step is to make sectional impressions of the upper and lower jaws and teeth. These are made in trays having no buccal or labial flange. We use compound again for the impression material. The teeth, after extraction, are set into their respective imprints in these sectional impressions and models are secured therefrom that will accurately reproduce the characteristic $\cdot$ features of that natural denture.

While making these preliminary stud- 
ies and measurements, and impressions we have discussed with the patient his several peculiar features and mutually agreed upon what features shall be reproduced and what ones altered or modified in the prosthetic denture. This is the time for the dentist to anticipate the necessary changes and inform his patient as to why such changes are necessary.

The selection of teeth for the case comes next.

In the selection of teeth, I find all patients reasonable when we have their own natural teeth as a guide. Ofttimes it is desirable to have another member of the patient's family or some close friend assist in the selection of teeth. Seldom does a case present that requires any special diplomacy at this stage.

With these preliminary steps complete, we are ready to proceed with the operation for removal of the teeth and preparation of the ridges to better receive our dentures.

\section{Extractron and Alveolotomy}

We will detail an application of the method to a case requiring removal of the maxillary teeth and alveolotomy and the making of a restoration to occlude with the natural mandibular teeth.

\section{ANESTHETIC}

The anesthetic of choice for this surgical operation is procaine employed in the nerve-blocking methods.

Any general anesthetic may put the patient into a condition that precludes our proceeding with denture construction immediately after the surgical work is done.

\section{Removal of Teeth and Alveolar Process}

When the parts are thoroly anesthetized, the teeth and adjacent soft tissues should be cleared of all foreign substances by using gauze or cotton sponges; sprays of antiseptic solution; washed over with $60 \%$ alcohol; and then painted with menthol-iodin solution.

In order that we may keep the parts in good condition while operating, it is advisable to divide the whole field into sections and operate upon them as consecutive steps. Do not attempt to cover too much territory at one step. It is better that we should take only four or five teeth for one step and do this much thoroly and in a cleanly manner rather than to attempt making a complete extraction before trimming any of the process.

When the teeth and adjacent soft tissues have been made ready for the operation, our first instrument used is the lance. Make a clean incision continuous along the labial or buccal gingival border of as many teeth as have been selected for the first step; then make a like incision in the linguo-gingival border.

These teeth are then carefully extracted. If any root fragments break off and remain in the process, do not attempt further to remove them now but wait until the later stage when the tissues are opened wide and ready access afforded.

In the next step, we carefully lift the labial and buccal mucoperiosteal tissue beginning at the incised gingival border, lifting it up from the process so far as we have predetermined the process should be removed.

What determines how much process should be removed?

Just two considerations :- contour and pathology.

You will remove no more than will permit the necks of the porcelain teeth to be placed in the desired position and permit a labial border for the denture; you will remove enough to obviate objectionable contours; you will remove whatever is necessary to eliminate pathology.

It is seldom ever necessary to disturb the lingual mucoperiosteum.

\section{Alveolotomy}

With suitable bone-cutting forceps or ronguers, we then remove the boney septa between the alveoli and then the labial or buccal plate of process to a distance 
extending from half to two-thirds the depth of the alveolus. Once in a while a case presents wherein it is necessary to go beyond the apex of the alveolus; but such cases are unusual.

At this stage of the work, we must be on the lookout for pathological conditions. We have X-ray films to guide us in this work and we now fail to be surprised to find conditions worse than the X-rays indicated.

Some operators make extensive use of chisels, others employ bone burs and stones for the removal of the process. I have had best success with a suitably shaped ronguer. 'With this instrument, we first bite out sections with a labio-lingual cut and then go over this edge with a mesio-distal cut. This mesio-distal cut is for the purpose of smoothing the alveolar rim, freeing it of any minor points or projections. The same trimming or smoothing may be accomplished with an antrum rasp or a heavy bone curette or bone burs or stones. Our preference is for the instrumentation that leaves the minimum of fragments and bone debris in the field of operation because we wish to avoid the necessity of irrigating the parts. Irrigating and flushing the field frees it from debris but, in our humble opinion, it is not beneficial to the tissues that are to remain.

With your first step or area completed to this stage, continue to the next and the next until the entire jaw is operated in the features described above.

The lingual border of process is likewise smoothed off but not reduced materially.

A study of many cases for months after the operation, with such changes as progressively come to some of them, convinces me that the surgeon who reduces the lingual alveolar borders errs in proportion as he reduces them. Some operators apparently forget that another dentist must follow them with denture construction when healing is complete, and their enthusiasm carries them so far in tissue removal that the result can be called by no other name than wanton destruction.

It has been our good fortune to be our own follow-up dentist so we have studied methods and results from both viewpoints.

One must not formulate the idea that this surgical technic will result in a foundation for a denture that positively will not change after the denture is worn for a short time, we have not attained that millennial state yet. Such a happy result seems to have been reached in perhaps 20 per cent of the cases operated but in the other 80 per cent from one to three relinings will be necessary within a year. To whatever degree pyorrhea is the cause of tooth loss, progressive atrophy of the ridges is to be expected.

Experience in relining has taught us that whatever change occurs will be evident in the buccal side of the ridge (and labial) and the lingual or palatial changes only enough to accommodate the falling crest of the ridge.

We would advise that in the operation of alveolotomy "the lingual rim of process should be merely smoothed off but not reduced materially."

The gum flaps, whose edges were freshened by the lance prior to extraction, are brought back to place and approximated. If any considerable quantity of process has been removed, the labial and buccal flap will be too long; it will overreach the lingual border. Observe the distance it overreaches and with surgical scissors remove the surplus tissue. At this stage, aim to prepare both flaps so that when sutured together they just reach each other nicely to close the wound in an even clean line, having neither a stretching nor a flabbiness of the labial flap.

Horsehair, used with the continuous suture, has been most satisfactory in our practice.

The several steps of surgical proce- 
dure thus hastily outlined offer us a great advantage in that we are now able to set the necks of our porcelain teeth into a position formerly occupied by the natural tooth neck; and furthermore we also gain room for a labial rim of vulcanite.

By the older methods, we would wait for months for the dissolution of this labial and buccal alveolar border and, even after patient waiting, be compelled at times to set the teeth in a rather forward position and dispense with the labial rim on our denture. As long as nature takes away this process ultimately, we might as well anticipate nature by removing it surgically, thus hastening the time when our patient can be comfortably equipped with artificial dentures.

\section{IMMEDIATE IMPRESSIONS}

When the surgical steps are completed, we take any one of the ordinary impression trays that will fit to the mouth so that the rim of the tray will reach well up to the reflex of the lip and cheek attachments and clear all the ridge tissue. In the heel, the tray must pass the tuberosities and extend slightly onto the soft palate. Thus it is a trifle larger in all dimensions than our finished dentures will be.

Dry the tray and warm it; then with stick compound lay on a bead of compound just inside the rim of the tray and extending entirely around the periphery labial, buccal, and heel. Dip into water heated to $160^{\circ}$ to soften the compound uniformly and pass into the mouth. Let the seating of the tray be guided solely by the middle finger held in the median line well back on the tray. The rim of soft compound comes into contact with the tissues above and beyond the operated areas and affords a wellfitted rim. A small roll of black carding wax such as our vulcanite teeth are mounted upon will serve the same purpose nicely and is readily adapted to the tray.

A hole is now drilled thru the vault of the tray with a No. 4 bur; a plaster wash is placed in the tray and carried back into place in the mouth, as in the Hall method. Our well fitted rim would make difficult the escape of surplus plaster as the tray approaches its maximum seating. This little hole in the vault of the tray affords a vent for the excess plaster.

As the impression is complete we then examine the vault with considerable pressure of the index finger to determine the location of any hard parts. These areas should be relieved by scraping the impression just made.

Paint the impression with separating medium and pour with plaster of Paris.

This impression and cast secured from it are only approximately correct; so it is permissible to use plaster of Paris and add potassium sulphate to hasten the setting.

In five minutes, we can shave off the impression plaster and recover the cast.

Over this preliminary cast, we warp a baseplate of material that will withstand the heat of the mouth.

For this purpose, S. S. W. Ideal baseplate has been found very satisfactory. Trim the plate to the approximate limits of the proposed denture.

Shape a piece of 14 gauge stiff wire to the vault contour and extending from tuberosity to tuberosity. Heat the wire and apply it to the hard baseplate, imbedding it in the wax. This wire stiffens the base and prevents its being warped in the later steps.

Carry this baseplate to the mouth and test it for rim heighth as accurately as can be done with open mouth inspection and determine that the length in the vault is satisfactory. We desire the baseplate rims to be about one millimeter lower buccally and labially than the finished denture; we desire the baseplate to extend four millimeters back onto the soft palate between the tuberosities.

Usually this baseplate will be sufficiently well fitted so that it will retain its position in the mouth unaided. 


\section{Re-establishing Central Occlusion}

Have the patient close the lips and swallow with the baseplate in the mouth, coming to a rest position of the lips and mandible when the act of swallowing is done. Following the Greene-Supple methods for re-establishing central occlusion, we direct the patient to retain this rest position of the mandible while we part the lips with our fingers and observe how much space is between the baseplate and the mandibular teeth. This space is to be filled with ordinary baseplate wax. Add this wax to the hard baseplate just as we ordinarily build up occlusion rims-except that for our present purposes we desire a little excess in the length occlusally.

The occlusal plane and length and fullness of wax form is now established in the mouth by the steps and procedure employed in the Supplee method of impression making.

Having first trimmed the wax form approximately with a knife, warm its occlusal surface to a slight depth; return into the mouth; have the patient close the lips and swallow.

If the expression of the lips is tense and drawn, have them swallow again and bite. Often this step must be repeated warming the occlusal surface of the wax before each effort and trimming off the surplus afterward, so that only the indentations of the occluding cusps are evident in the occlusal surface of the wax. Be sure at all times to maintain a flat occlusal surface in the wax.

By reference to the record of our preliminary measurements and to the study casts, we can now determine to a nicety when we have reestablished the desired central occlusion and fullness of contour. Remember that the patient's upper lip is not a safe guide just now for it is still anesthetized and is longer and thinner than normal. This fact emphasizes the necessity of having some other guide such as is afforded by our records previously made.

When satisfied with the re-established relation of maxilla and mandible and fullness of contour, we are ready to proceed with the setting in of the teeth.

In arranging the teeth, we begin with the first molar on one side, cutting out enough wax to allow it to set into position; then set the opposite first molar.

As these teeth are put into approximate position one by one, we return the baseplate to position in the mouth and bring the mandibular teeth into central occlusion. This enables us to place each tooth most advantageously.

Following the first molars, next place the bicuspids and then the second molars, if the space is ample. If crowded for space, we grind and set the second molars after all the other teeth are in place.

With the bicuspids in place on both sides, it is time to proceed with the front six teeth, arranging them to suit the space," occluding teeth, and aesthetic sense of yourself and patient.

As each tooth is arranged to satisfaction, we have found it advisable to secure it in place with some harder wax, like Kerr's setting-up wax. This gives more assurance that the arrangement will not be spoiled while setting subsequent teeth.

All the teeth are now in position on the wax base plate and we wax up the lingual practically to a finished model and wax the labial and buccal so that the desired contour will be complete almost to the rim of the baseplate.

When necessary our baseplate may now be post-dammed by means of a little roll of black carding wax applied across the heel, warmed, and pressed to position in the mouth. This insures a perfect seal in this part of the denturea thing just as important as perfect rim fit thru the balance of the periphery of the denture.

At this stage, we resort to the plaster of Paris wash again. We convert our base plate with the teeth set up on it, 
made from an open mouth impression, into an impression tray with which we secure a closed mouth impression under slight occlusal stress. .

From this point forward to completion, the procedure is nothing different from that which any good, careful denture technic teaches.

Trim the plaster impression from tuberosity to tuberosity just as you desire the finished denture to be; we pour this impression with Brophy's Artificial Granite which is a material that sets as quickly as plaster and which is claimed to be free from expansion or contraction. Complete the waxing, having first removed any excess plaster from lingual surface and outer rims; flask, pack, vulcanize and finish "as all good dentures should be."

Before dismissing the patient, one further precaution can be taken to prevent a damaging collapse of the temporomandibular articulation. Warp another baseplate on your approximate cast, reinforcing it with wire across the heel. Now build the occlusion rim with modeling compound instead of wax-and you have thus made something which your patient may wear several days (if necessary); and I have known patients to chew food on this base.

You have given your patient a denture that he can wear today and maybe for months or years with now and then the usual trimming to accommodate new pressures and stresses as he becomes more and more accustomed to the new device.

You have not compelled him to go about for weeks with a wrecked articulation which results always in a damaging of the capsular ligament in an effort to rest the mandible or masticate food; you have relieved him of much misery that is suffered because of cramping of the muscles when without teeth; you have obviated the forming of the bad habit of protruding the mandible in masticating -a habit most edentulous patients acquire in a very short time.
Your patient after just a few hours of toothless retirement, can appear again and attend his business or social duties without embarrasment. Your patient will not suffer as much from sore gums while wearing this denture as he would without it and in a few days he will have a better ridge than he would have had under old methods after waiting weeks or months.

The expressions of comfort that the patient experiences are most gratifying. Nearly every patient after 24 hours is convinced that he is more comfortable with than without and right there the greatest difficulty is passed for the denture prosthetist. Whenever your patient realizes that his personal comfort is increased by keeping the denture in his mouth, that patient will readily learn to use the denture.

Please bear in mind also that the patient has not had time to forget how to use teeth; normal mandibular control has not been lost; improper habits of mandibular movements have not been formed. The transition from natural denture to prosthetic denture is surprisingly easy.

For yourself, you have eliminated the use of any articulator except the one best one, which is the patient's own temporomandibular joint. On it, you can open or shut the maxillo-mandibular relation without fear of creating an incompatible relation between articulation and occlusion. You can make an arrangement of teeth that is harmonious in all respects and a more accurate duplication of nature's organs both as to form and position.

Oliver Wendell Holmes said: "The dental profession has embellished and prolonged the reign of beauty. It has added to the charm of social intercourse. It has lent perfection to the strain of eloquence-and has taken from old age its most unwelcome feature."

The methods above outlined have obviated that "most unwelcome feature" from all ages. 